\title{
Aceite residual automotriz como aditivo en mezclas de hormigón: Si es factible su uso
}

\author{
Automotive waste oil as an additive \\ in concrete mixtures: its use is feasible
}

Trino Baloa Montilla ${ }^{1}$, Carlos Arellano Mogollón ${ }^{2}$, Juan De Abreu Gómez ${ }^{2}$, Idalberto Aguila Arbolaez ${ }^{3}$

\footnotetext{
${ }^{1}$ Instituto de Materiales y Modelos Estructurales, Universidad Central de Venezuela, Caracas, Caracas Venezuela.

${ }^{2}$ Escuela de Ingeniería Civil, Universidad Central de Venezuela, Caracas, Caracas Venezuela.

${ }^{3}$ Instituto de Desarrollo Experimental de la Construcción, Universidad Central de Venezuela, Caracas, Caracas ,Venezuela.

e-mail: trino.baloa@ucv.ve
}

\section{RESUMEN}

El encontrar un uso al Aceite Residual Automotriz (ARA) es preponderante para la sociedad, ya que, un litro de este líquido es capaz de contaminar un millón de litros de agua potable, sin contar los daños a suelos agrícolas y aguas subterráneas. El presente trabajo tiene por objeto estudiar las propiedades físicas y mecánicas del hormigón al incorporar ARA en dosificaciones entre $0,10 \%$ y $0,80 \%$ por peso de cemento. Para alcanzar este objetivo se obtuvieron asentamientos de las mezclas frescas y las resistencias del hormigón endurecido para diferentes dosificaciones de ARA. De las resistencias obtenidas a los 28 días, se presenta una curva de regresión polinómica que permite obtener un valor óptimo de ARA, éste valor es utilizado en los ensayos de contenido de aire y tiempo de fraguado para el hormigón fresco. Mientras que para el hormigón endurecido se realizaron ensayos de resistencias a la compresión, a la tracción, velocidad de pulso ultrasónico, absorción y adherencia desarrollada entre el hormigón y las barras con resaltes. Los resultados indican que dosificaciones de ARA entre $0,10 \%$ y $0,30 \%$, aportan mayor resistencia a la compresión del hormigón, siendo la dosis óptima de $0,14 \%$. Al utilizar la dosis óptima de ARA se validó que todos menos dos de los ensayos realizados en este estudio son similares al hormigón de control, las excepciones fueron la resistencia a la compresión que aumenta en un $8 \%$ y la adherencia desarrollada entre el hormigón y, las barras con resaltes que disminuye en un $9 \%$. Por tanto, se concluye que el uso de ARA como aditivo en el hormigón, al 0,14\% por peso de cemento, es factible en las mezclas de hormigón no estructural, específicamente en brocales, pavimentos rígidos, aceras, bloques, adoquines y senderos peatonales. Siendo el uso del ARA como aditivo, un aporte significativo para reducir el daño que genera al ecosistema.

Palabras Clave: aceite residual automotriz, adherencia, aditivo, barra de refuerzo, hormigón.

\section{ABSTRACT}

Finding a use for Automotive Residual Oil (ARO) is preponderant for society, since one liter of this liquid is capable of contaminating one million liters of drinking water, without counting the damages to agricultural soils and groundwater. The purpose of this work is to study the physical and mechanical properties of concrete by incorporating ARA in dosages between $0.10 \%$ and $0.80 \%$ by weight of cement. To achieve this goal, settlements of fresh mixtures and resistances of hardened concrete were obtained for different dosages of ARO. From the resistances obtained after 28 days, a polynomial regression curve is presented that allows obtaining an optimum value of ARO, this value is used in the air content and setting time tests for fresh concrete. While for the hardened concrete tests were carried out resistance to compression, tensile, ultrasonic pulse speed, absorption and adhesion developed between concrete and bars with projections. The results indicate that dosages of ARO between $0.10 \%$ and $0.30 \%$, provide greater resistance to compression of the concrete, with an optimum dose of $0.14 \%$. When using the optimal dose of ARO it was validated that all but two of the tests carried out in this study are similar to the control concrete, the exceptions were the resistance to 
compression that increases by $8 \%$ and the adhesion developed between the concrete and, bars with projections decreases by $9 \%$. Therefore, it is concluded that the use of ARO as an additive in concrete, at $0.14 \%$ by weight of cement, is feasible in non-structural concrete mixtures, specifically in curbs, rigid pavements, sidewalks, blocks, pavers and paths pedestrian Being the use of the ARO as an additive, a significant contribution to reduce the damage it generates to the ecosystem.

Keywords: automotive residual oil, adhesion, additive, rebar, concrete.

\section{INTRODUCCIÓN}

Las construcciones de obras civiles en hormigón reforzado son utilizadas a nivel mundial. Primero por la nobleza del hormigón para ser manipulado y moldeado según convenga, segundo, la facilidad que tiene el ser humano de construir con este material. Respecto al uso de residuos, la industria de la construcción es una de las más útiles para reutilizar o en otras ocasiones eliminar pasivos ambientales que son generados por diferentes industrias. Los antecedentes que se han encontrado acerca del Aceite Residual Automotriz (ARA) son de mucha atención, ya que, este residuo afecta suelos fértiles, ríos, mares y hasta el aire, al ser utilizado como combustible propiciando un daño al medio ambiente. Tanto, que un litro del ARA contamina directamente a más de un millón de litros de agua potable.

EL-FADEL et al. [1] describe las prácticas de gestión que se realiza a los residuos de los aceites provenientes de la industria petrolera del Líbano, identificando los posibles impactos ambientales adversos de no realizarse dichas prácticas. Asimismo, proponen estrategias para el manejo adecuado de residuos por parte de la sociedad incrementando la conciencia ambiental. Dentro de las estrategias se estudia como opción de gestión, la viabilidad económica del reciclaje de residuos de aceite. Otra alternativa para la disminución de contaminantes, la presentan TORRES et al. [2], estos investigadores reportan resultados satisfactorios para neutralizar un material contaminante de la petroquímica utilizando técnica de solidificación y encapsulamiento de los metales pesados por medio de la hidratación del cemento Portland. Para validar la fijación de los contaminantes en la matriz cementante utilizaron la técnica de lixiviación "Toxicity Characteristic Leaching Procedure" en morteros de cemento con adición de hasta un $20 \%$ del residuo petroquímico, el cual, contiene elementos químicos tales como el $\mathrm{As}, \mathrm{Pb}, \mathrm{Zn}, \mathrm{Cr}$ y La. Luego de aplicar la técnica de lixiviación se comprobó que los elementos contaminantes no alcanzaron los valores máximos permisibles.

Otros estudios a nivel mundial se han realizado respecto a la reutilización de residuos para la industria cementera y de la construcción. GARCÍA et al. [3] indican que muchos residuos pueden ser empleados en tres fases diferentes del proceso productivo: en la preparación inicial, como correctores de las materias primas y/o como combustible alternativo, como adiciones activas al clinker Portland y como árido de reciclado en bases y sub bases de carreteras y en la fabricación de hormigón. PELLISER et al. [4] Este trabajo presenta el potencial de utilizar tiras de gomas recicladas en matrices cementantes. Esto contribuye al desarrollo de construcciones sustentables. Las dosificaciones para realizar el hormigón implican el reemplazo del agregado de arena por tiras de goma convencional, además, de goma modificada con adición de humo de sílice y activación alcalina. La caracterización del hormigón se realizó aplicando ensayos de resistencia a la compresión, módulo de elasticidad, densidad y microestructura. Los autores indican que el reciclar las tiras de goma promete ser un excelente componente como agregado del hormigón. Esto se observó al solo reducirse la resistencia a la compresión en un 14\% a los 28 días de edad, en comparación con un hormigón convencional de alta resistencia de $48 \mathrm{Mpa}$. El hormigón puede soportar la construcción sostenible, debido a que se reducen el consumo de recursos naturales por el uso de residuos industriales y la producción de un material especial. TANGCHIRAPAT et al. [5] La ceniza de las cascaras del aceite de palma se producen al utilizarlas como combustible de las calderas en la industria del aceite comestible de la palma real africana, estas cenizas se disponen es patios de residuos. Los autores utilizan esta ceniza como puzolana en el hormigón agregando, ceniza original como sale de la zona de combustión (OP), otra ceniza con tamaño de granos de 15,9 micras (MP) y de tamaños de granos de 7,4 micras (SM). Se reemplaza el cemento Portland tipo I es reemplazado por OP, MP y SP en 10\%, 20\%, 30\% y 40\% por peso de cemento. Se estudiaron la resistencia a la compresión y la expansión debido al ataque de sulfato de magnesio. Los resultados indican que OP no que las resistencias son menores al cemento Portland tipo I. Mientras que las sustituciones de MP y SP en $20 \%$ y $30 \%$, alcanzan resistencias a la compresión mayores a la edad de 90 días y una expansión similar a la del cemento Portland tipo I a los 365 días de estar sumergidas las mezclas antes mencionadas, en un solución de 5\% de sulfato de magnesio. PAPPU et al. [6]. Los autores indican la producción de residuos sólidos en la India para el año 2007. Presentan cifras aproximadas de 960 millones de toneladas de residuos sólidos que se generan anualmente en la industria, minería, municipios, agricultura y otros procesos. El desarrollo de la gestión de los residuos sólidos a resultado en materiales alternativos para la construcción al 
sustituir materiales tradicionales en componentes de bloques, alambres, agregados, cerámicas, cemento, suelos y pinturas. En definitiva, presentan el estatus en la generación y utilización de residuos sólidos riesgosos y no riesgosos en la India; estos potenciales residuos reciclables son reportados y discutidos en detalle. FRÍAS et al. [7] manifiesta que el uso de materiales que son susceptibles de ser empleados como adiciones o aditivos, no sólo es una contribución parcial a la disminución del impacto ambiental y ahorro energético, sino que además pueden mejorar sus propiedades, tales como la resistencia y durabilidad en el hormigón. En específico para esta investigación, el estudio del aceite de motor residual en el hormigón es primordial. BILAL y AHMAD et al. [8] presentan resultados donde indican que el aceite de motor usado actuó como un agente que incorpora aire mejorando el asentamiento y la fluidez de la mezcla de hormigón. Las reducciones en la resistencia a compresión del hormigón debido a la incorporación de aceite no fueron tan significativas como cuando se utilizó un aditivo químico comercial incorporador de aire. En Malasia, NASIR et al. [9] presentan resultados de un estudio experimental acerca de los efectos de las propiedades en el hormigón fresco y endurecido al incorporar aceite de motor residual, obteniendo un incremento en los asentamientos de la mezcla de hormigón fresco entre un 18\% y 38\%, un contenido de aire ocluido entre $26 \%$ y $58 \%$. Además, al estudiar la porosidad y permeabilidad del oxígeno en el hormigón endurecido se obtiene una reducción de estas propiedades en hormigón con aceite de motor residual manteniéndose similares las resistencias a la compresión con respecto a las mezclas de control. En este estudio se realizan procedimientos establecidos en las normativas ASTM para comprobar la factibilidad del uso del aceite residual automotriz como aditivo en mezclas de hormigón.

\section{MATERIALES, DOSIS, MEZCLAS Y MÉTODOS}

\subsection{Materiales}

Aceite Residual Automotriz (ARA): aceite usado del tipo monogrado para motores Diesel proveniente de la flota de camiones mezcladores de la Fábrica Nacional de Cemento, planta concretera San Antonio de El Valle, Caracas.

Cemento Portland tipo I-R: fue suministrado por la planta de la Fábrica Nacional de Cemento (FNC) de Ocumare del Tuy, Miranda.

Áridos: La arena es de sílice y natural de río proveniente de la Arenera El Carmen, estado Miranda. Posee una granulometría bien gradada con un peso específico de 2,71. Pesos unitarios sueltos y compactos de $1,50 \mathrm{~kg} / \mathrm{m}^{3}$ y $1,61 \mathrm{~kg} / \mathrm{m}^{3}$, respectivamente. La piedra picada es de caliza con tamaño máximo de 1 " y proviene de la cantera Cura, estado Carabobo. Posee una granulometría bien gradada con un peso específico de 2,65. Pesos unitarios sueltos y compactos de $1,51 \mathrm{~kg} / \mathrm{m}^{3}$ y $1,68 \mathrm{~kg} / \mathrm{m}^{3}$, respectivamente.

Barra de acero con resaltes (cabilla): proveniente de la industria Siderúrgica del Turbio (SIDETUR) con denominación SV4S60, es decir, barra de acero de media pulgada, no soldable con una resistencia cedente de $4.200 \mathrm{kgf} / \mathrm{cm}^{2}$.

\subsection{Dosificaciones}

La resistencia a la compresión esperada a los 28 días (f'c) es de $210 \mathrm{kgf} / \mathrm{cm}^{2}$, cuya resistencia promedio para el cálculo de las dosis de los componentes del hormigón es de $315 \mathrm{kgf} / \mathrm{cm}^{2}$. Vale destacar, que la técnica empleada en este estudio para obtener las cantidades de los componentes de la mezcla de hormigón se obtuvo a partir de los diseños experimentales realizados en el Instituto de Materiales y Modelos Estructurales (IMME) y compilados en PORRERO et al. [10] combinado con GARCÍA [11]. Vale destacar que las cantidades están en kilogramos, siendo la cantidad de ARA, el producto del peso de cemento por cada uno de los porcentajes que se presentan en la tabla 1. Adicionalmente, ésta tabla presenta la dosis de los componentes utilizados para elaboración del hormigón. 
Tabla 1: Dosis de los componentes para hormigón $\mathrm{f}^{\prime} \mathrm{c}=210 \mathrm{kgf} / \mathrm{cm}^{2}$.

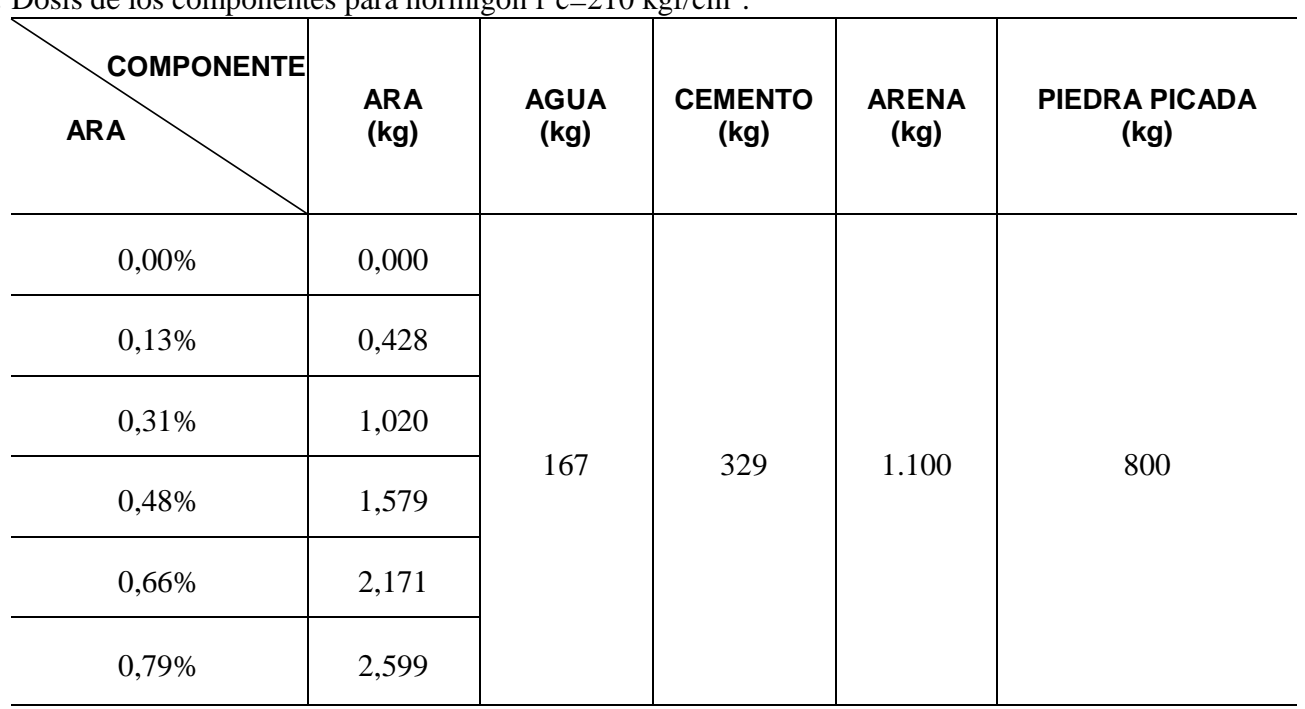

\subsection{Mezclado, moldeo y curado}

Para la elaboración de las mezclas, moldeo y curado se utilizó la norma ASTM C192. Las probetas cilíndricas de hormigón de $10 \mathrm{~cm}$ x $20 \mathrm{~cm}$ (diámetro x altura) son utilizadas para la compresión simple, luego se aplica un factor de corrección por tamaño de probetas de 0,98 para correlacionar valores con probetas cilíndricas normativas de $15 \mathrm{~cm}$ x $30 \mathrm{~cm}$ (diámetro x altura). Vale resaltar que este factor se obtiene RAMOS [12] a partir de la elaboración de un mínimo 30 mezclas con 3 réplicas por mezcla y ensayadas a compresión a 3 edades (7 días, 14 días y 28 días). Los ensayos a tracción indirecta, cambios bruscos de temperatura, ultrasonido, absorción y el de adherencia del acero con el hormigón se utilizan probetas cilíndricas de hormigón de $15 \mathrm{~cm}$ x $30 \mathrm{~cm}$ (diámetro x altura), mientras que para el ensayo de tracción a la flexión, se utilizan probetas prismáticas de sección transversal de $15 \mathrm{~cm}$ x $15 \mathrm{~cm}$ y longitud de $45 \mathrm{~cm}$. El curado de las probetas se llevó a cabo una vez pasadas las 24 horas de endurecimiento de las probetas, las cuales, son retiradas de la piscina de curado en la edad fijada para el ensayo específico (1 hora antes de ensayar).

\subsection{Métodos}

En principio, se realizan ensayos de caracterización física del ARA y de un mismo tipo de aceite pero sin usar (Maxidiesel plus SAE 50). Específicamente, se aplicaron métodos para la determinación de la densidad relativa, punto de inflamación, contenido de agua y de sólidos, número base y número ácido. Todos estos ensayos son sugeridos BARRAGAN y BOHORQUEZ [13], bajo las consideraciones de las normas ASTM, las cuales, se indican en la tabla 2.

Tabla 2: Normativas para caracterizar el ARA.

\begin{tabular}{c|l}
\hline ASTM D 2896-07a & $\begin{array}{l}\text { Standard Test Method for Base Number of Petroleum Products } \\
\text { by Potentiometric Perchloric Acid Titration. }\end{array}$ \\
\hline ASTM D 974-14e1 & $\begin{array}{l}\text { Standard Test Method for Acid and Base Number by Color- } \\
\text { Indicator Titration. }\end{array}$ \\
\hline ASTM D 56 & Standard Test Method for Flash Point by Tag Closed Cup Tester. \\
\hline ASTM D 1298 & $\begin{array}{l}\text { Standard Test Method for Density, Relative Density, or API } \\
\text { Gravity of Crude Petroleum and Liquid Petroleum Products by } \\
\text { Hydrometer Method. }\end{array}$ \\
\hline ASTM D 95 & $\begin{array}{l}\text { Standard Test Method for Water in Petroleum Products and Bi- } \\
\text { tuminous Materials by Distillation. }\end{array}$ \\
\hline
\end{tabular}

Posteriormente, se inició el mezclado de 3 réplicas por cada una de las 3 mezclas realizadas para las edades de 7 días, 14 días y 28 días. Cabe resaltar que estas mezclas cumplieron con las dosificaciones presen- 
tadas en la Tabla 1 (incorporación de ARA por peso de cemento en $0,00 \% ; 0,13 \% ; 0,31 \% ; 0,48 \% ; 0,66 \%$ y $0,79 \%$ ). Luego, con dichos resultados se desarrolla una gráfica de resistencia a la compresión contra porcentaje de ARA, la cual, permitirá obtener por medio de regresión polinómica el porcentaje óptimo de ARA a ser incorporado en las mezclas de hormigón. Los ensayos realizados junto a la metodología que guío el estudio, están contenidas en las ASTM, las cuales se presentan en la Tabla 3.

Tabla 3: Métodos que rigen ensayos realizados en este estudio.

\begin{tabular}{c|l}
\hline ASTM C 143 / C143M-15a & $\begin{array}{l}\text { Standard test method for slump of hydraulic-cement con- } \\
\text { crete }\end{array}$ \\
\hline ASTM C 403 / C403M-16 & $\begin{array}{l}\text { Standard test method for time of setting of concrete mix- } \\
\text { tures by penetration resistance. }\end{array}$ \\
\hline ASTM C 192 / C192M-2000 & $\begin{array}{l}\text { Standard practice for marking and curing concrete test } \\
\text { specimens in the laboratory. }\end{array}$ \\
\hline ASTM C 39 / C39M-17a & $\begin{array}{l}\text { Standard test method for compressive strength of cylin- } \\
\text { drical concrete specimens. }\end{array}$ \\
\hline ASTM C 496 / C496M-11 & $\begin{array}{l}\text { Standard test method for splitting tensile strength of cy- } \\
\text { lindrical concrete specimens. }\end{array}$ \\
\hline ASTM C 78 / C78M-2009 & $\begin{array}{l}\text { Standard test method for flexural strength of concrete. } \\
\text { (Using simple beam with load in the thirds of the light) }\end{array}$ \\
\hline ASTM C 642 / C642M-1997 & $\begin{array}{l}\text { Standard Test Method for Density, Absorption, and Voids } \\
\text { in Hardened Concrete. }\end{array}$ \\
\hline ASTM C 234 / C234-91a & $\begin{array}{l}\text { Standard test method for comparing concretes on the ba- } \\
\text { sis of the bond developed with reinforcing steel. }\end{array}$ \\
\hline ASTM C 597 / C597M-16 & Standard test method for pulse velocity through concrete. \\
\hline ASTM C 231 / C231-14 & $\begin{array}{l}\text { Standard test method for air content of freshly mixed } \\
\text { concrete by the pressure method. }\end{array}$ \\
\hline
\end{tabular}

\section{RESULTADOS}

\subsection{Caracterización física del Aceite Residual Automotriz (ARA)}

Para la caracterización física del ARA y el aceite sin usar se aplicaron los 5 ensayos según métodos presentadas en la Tabla 2. Los resultados obtenidos indican que hubo cambios significativos en el ARA al ser comparado con el lubricante sin usar, tal como se presenta a continuación en la tabla 4.

Tabla 4: Resultados de caracterización física del ARA y el lubricante sin usar.

\begin{tabular}{l|c|c}
\hline \multicolumn{1}{c|}{ ENSAYO } & $\begin{array}{c}\text { ACEITE RESIDUAL } \\
\text { AUTOMOTRIZ }\end{array}$ & $\begin{array}{c}\text { ACEITE SIN USAR O } \\
\text { VIRGEN }\end{array}$ \\
\hline Número Base (mg). ASTM D 2896-07a & $11,3 \pm 0,2$ & $6,5 \pm 0,8$ \\
\hline Número Ácido (mg). ASTM D 974-14e1 & $0,30 \pm 0,10$ & $2,12 \pm 0,01$ \\
\hline Contenido de agua (\%). ASTM D 95 & $0,41 \pm 0,01$ & $0,05 \pm 0,01$ \\
\hline Densidad (gr/cm3). ASTM D 1298 & $0,882 \pm 0,001$ & $0,897 \pm 0,003$ \\
\hline Punto de inflamación $\left({ }^{\circ} \mathrm{C}\right)$. ASTM D 56 & $218 \pm 10$ & $225 \pm 8$ \\
\hline
\end{tabular}

Vale destacar que tanto la alcalinidad del ARA como el contenido de agua aumentan en un $74 \%$ y $820 \%$, respectivamente. Al mismo tiempo que la acidez, la densidad y el punto de inflamación disminuyen en un $86,00 \% ; 1,67 \%$ y $3,11 \%$, respectivamente.

\subsection{Obtención de la dosis óptima de aceite residual automotriz}

\subsubsection{Asentamientos con relación de agua y cemento fija}

El procedimiento de la ASTM C 143 fue aplicado para calcular los asentamientos en el hormigón fresco de control y de las diferentes dosis de ARA por peso de cemento $(0,13 \%, 0,31 \%, 0,48 \%, 0,66 \%$ y 0,79\%). El diseño realizado fue para un hormigón de asentamiento 5". Siendo la dosis más favorable de ARA de $0,13 \%$ 
para un asentamiento promedio de 6,25" mientras que la mezcla de control es de 5,67". El resto de las dosificaciones de ARA están dentro de lo esperado pero por debajo de la mezcla control. La figura 1 presenta las curvas de asentamientos promedios y sus desviaciones estándar de las mezclas con diferentes dosis de ARA junto con la mezcla de control.

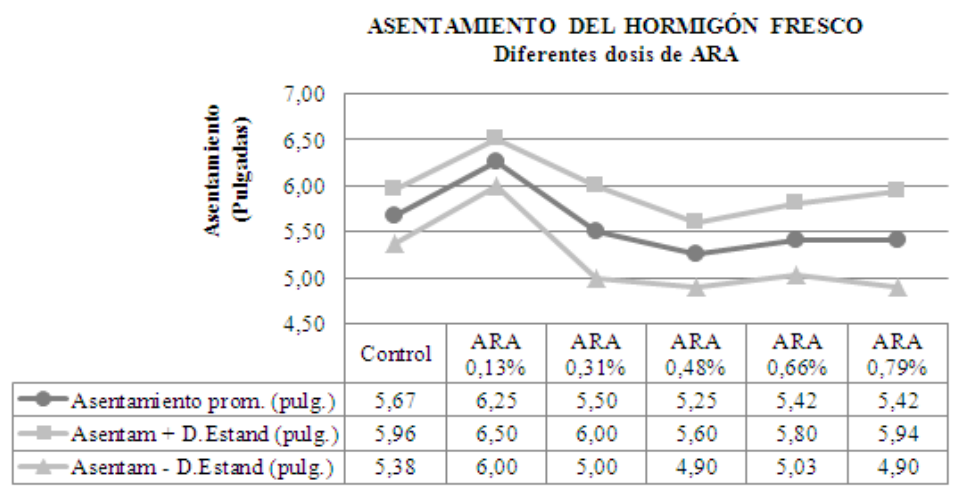

Figura 1: Asentamientos en el hormigón fresco con diferentes dosis de aceite residual automotriz.

\subsubsection{Resistencia a la compresión para obtener dosis óptima de ARA}

Siguiendo las consideraciones de la ASTM C 39, en la Figura 2 se presenta una gráfica con los resultados de la resistencia a compresión de las 3 edades de las probetas de hormigón que fueron ensayadas (7 días, 14 días y 28 días). En esta se observa que las mezclas de los hormigones que les fueron incorporados el ARA en proporciones entre $0,13 \%$ y $0,31 \%$, alcanzaron resistencias a la compresión por encima de la resistencia del hormigón de control.

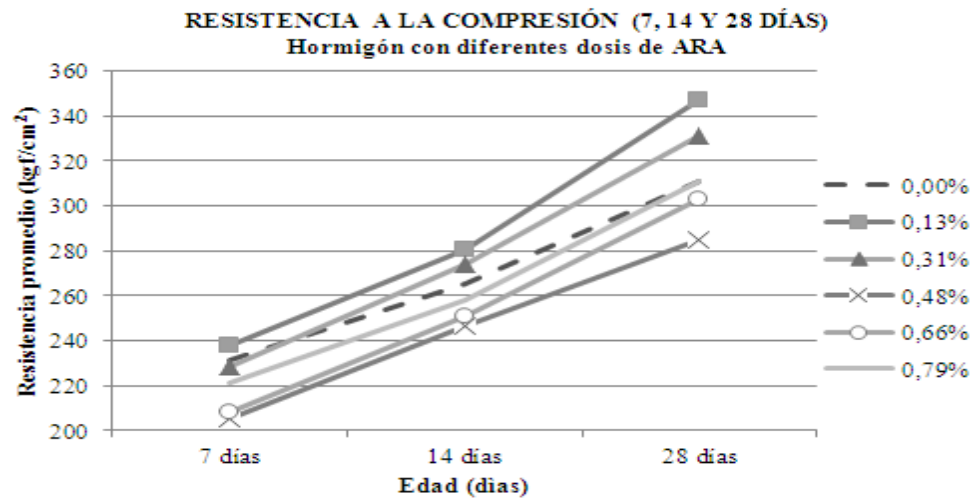

Figura 2: Resistencias a compresión de acuerdo a la dosificación y edad de las probetas.

De los valores de resistencia obtenidos con las dosificaciones de ARA incorporadas en el hormigón a la edad de 28 días, se elabora una curva de tendencia del tipo regresión polinómica de cuarto grado presentada en la figura 3 , cuyo coeficiente de determinación $\mathrm{R}^{2}=0,93$, aproximadamente. La dosis óptima de ARA obtenida fue de $0,14 \%$ para una resistencia estimada a la compresión de $352 \mathrm{kgf} / \mathrm{cm}^{2}$, mientras que la resistencia a la compresión del hormigón de control es de $311 \mathrm{kgf} / \mathrm{cm}^{2}$. 


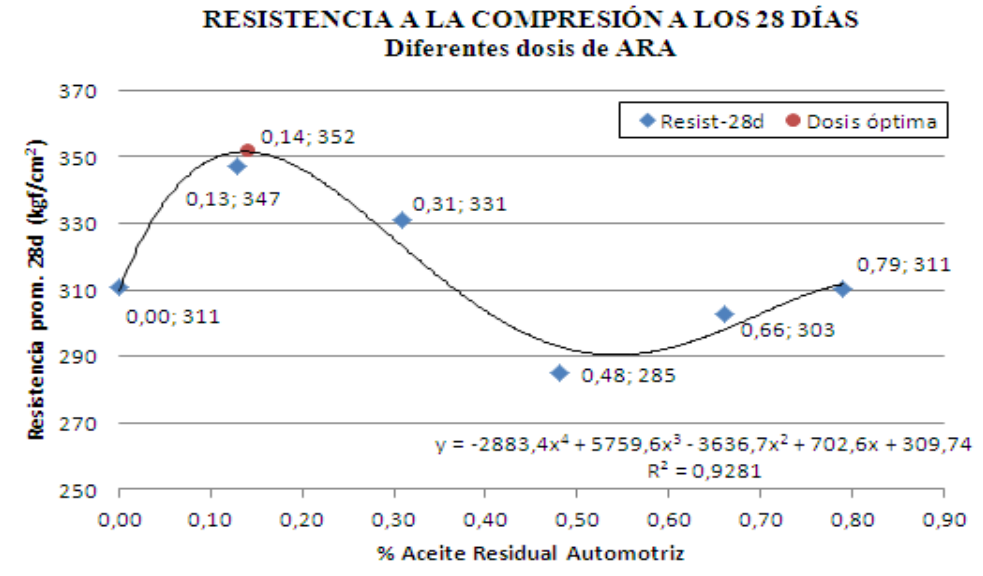

Figura 3: Resistencias a compresión de acuerdo a la dosificación y edad de las probetas.

Por tanto, esta dosis óptima de ARA es la empleada para obtener el hormigón en estado fresco y endurecido con el que se realizaran los ensayos normativos previstos en este estudio, tales como contenido de aire, tiempo de fraguado, resistencias a la compresión, a la tracción indirecta y por flexión, cálculo de velocidad de pulso ultrasónico, absorción y la adherencia entre el acero de refuerzo con el hormigón estructural.

\subsection{Estado fresco de Hormigón con dosis óptima de ARA}

\subsubsection{Contenido de aire}

Después de aplicar el procedimiento de la ASTM C 231, se indica que la dosis óptima de ARA alcanza $1,11 \%$ de contenido de aire, mientras que el hormigón de control es de 0,83\%. Es decir, ambos hormigones se encuentran dentro del rango aceptable de contenido de aire por no exceder el 3\%. Sin embargo, se evidencia que el ARA incorporó hasta un 34\% aunado a que la variabilidad en ambas mezclas es similar de $0,06 \%$. En la figura 4 se presenta un gráfica de barras con los valores antes mencionados de las mezclas frescas de los hormigones de control y con la dosis óptima de ARA.

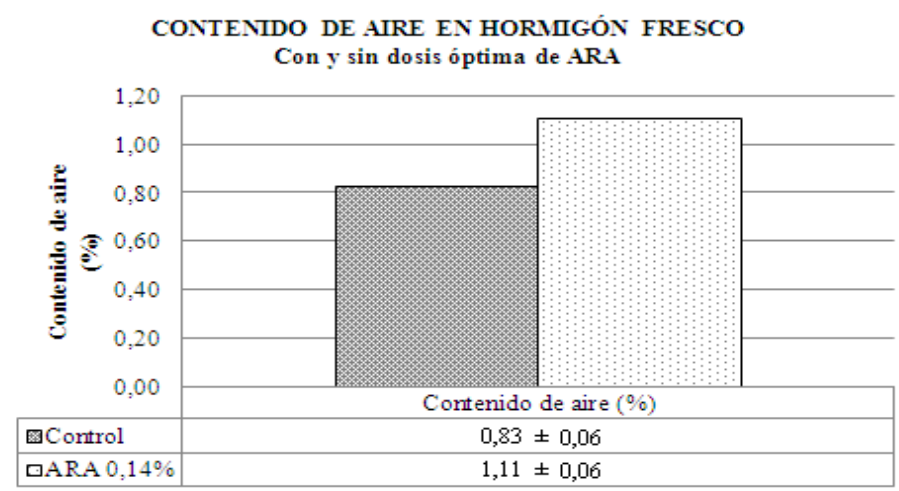

Figura 4: Contenido de aire en el hormigón fresco con y sin dosis óptima de aceite residual automotriz.

\subsubsection{Tiempo de fraguado}

Una vez realizado el procedimiento indicado en la ASTM C403, se obtiene que el hormigón fresco de control y el hormigón que le fue incorporado el ARA tienen un tiempo de fraguado inicial mayor a 4 horas con 45 minutos y un tiempo final de fraguado de 6 horas con 5 minutos. La figura 5 y 6 presentan las curvas de tendencia del tipo regresión exponencial, las cuales, intersecan a 2 rectas horizontales que indican los tiempos de fraguado inicial y final. 


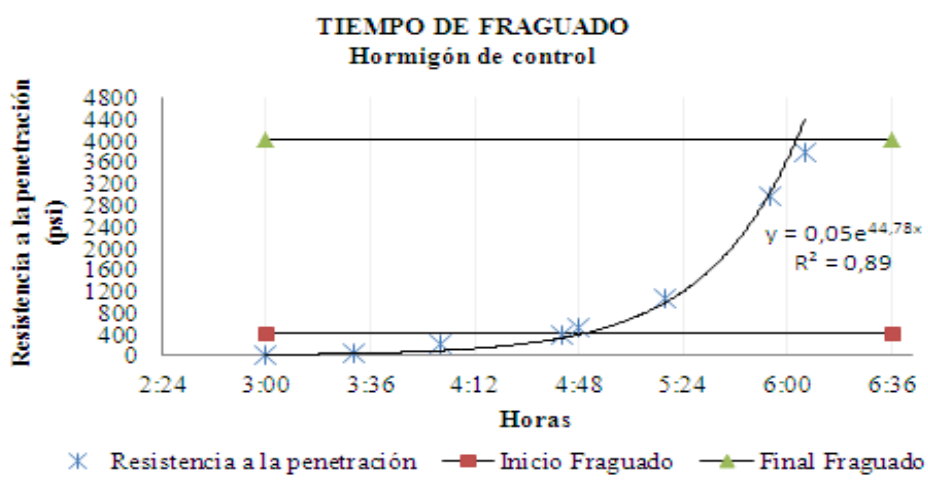

Figura 5: Tiempo de fraguado inicial y final del hormigón de control.

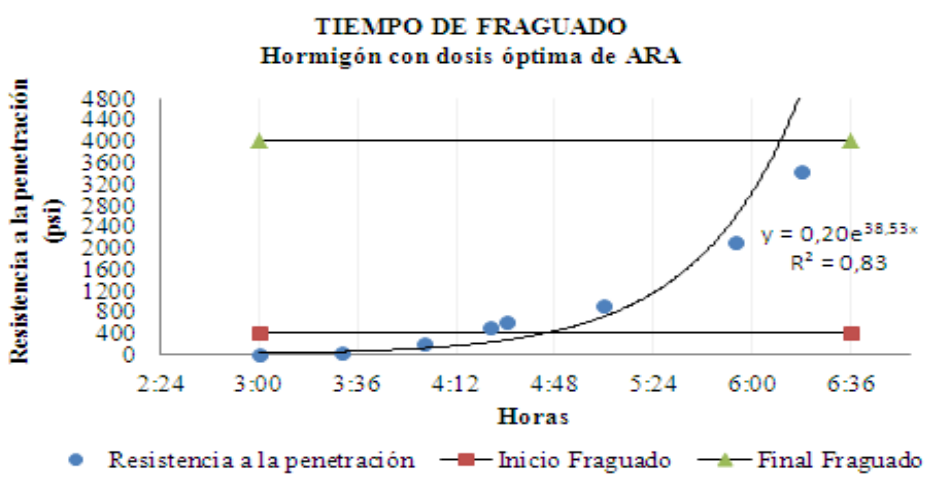

Figura 6: Tiempo de fraguado inicial y final del hormigón con dosis óptima de ARA.

El tiempo inicial de fraguado se obtiene del punto que se genera al intersecarse la curva de regresión exponencial ascendente con la recta horizontal que indica una resistencia a la penetración de 400 psi, mientras que el tiempo final de fraguado se obtiene del punto que se genera al intersecarse la curva de regresión exponencial ascendente con la recta horizontal que indica una resistencia a la penetración de 4000 psi. Realizando la diferencia del tiempo final (6 horas con 5 minutos, aproximadamente) y el tiempo inicial (4 horas con 45 minutos, aproximadamente) se obtiene que el tiempo de fraguado del hormigón de control y de dosis óptima de ARA es de 2 horas con 20 minutos.

\subsection{Estado endurecido del hormigón con dosis óptima de ARA}

En la preparación y curado de toda la probetas de hormigón que son utilizadas para los ensayos de resistencia a la compresión, resistencia a la tracción indirecta, resistencia a la tracción por flexión, absorción, velocidad de pulso ultrasónico y adherencia se consideró la ASTM C 192.

\subsubsection{Resistencia a la compresión (Control y dosis óptima de ARA)}

Siguiendo las consideraciones de la ASTM C 39, se obtuvieron valores de resistencia a la compresión de la mezcla control y de mezcla con $0,14 \%$ de ARA por peso de cemento. La Figura 7 presenta los resultados obtenidos a los 7 días, 14 días y 28 días. Vale destacar que las resistencias a la compresión del hormigón con dosis óptimas de ARA son mayores a la resistencia de control en las edades 7 días, 14 días y 28 días en un $9 \%, 13 \%$ y $8 \%$, respectivamente. 


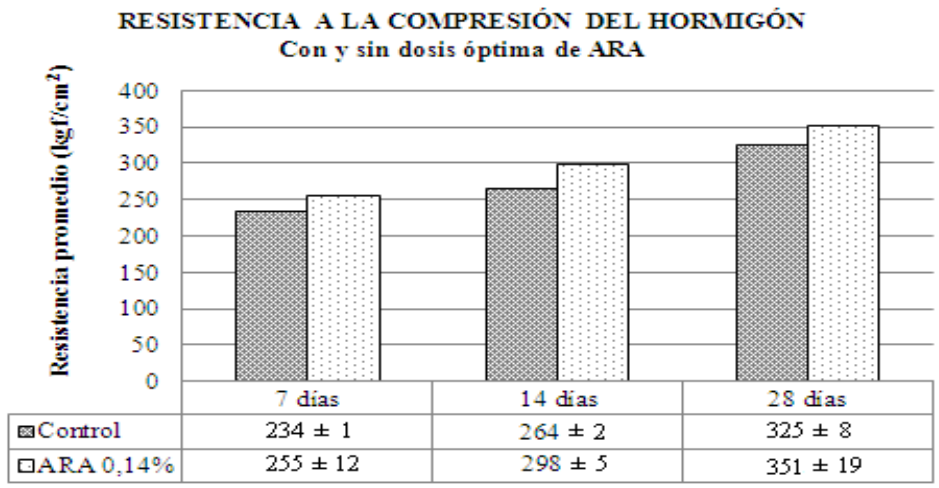

Figura 7: Resistencia a la compresión de hormigón con y sin dosis óptima de ARA.

\subsubsection{Resistencia a la tracción indirecta. (Ensayo brasilero)}

Del procedimiento propuesto en la ASTM C 496, se realizaron el ensayo de resistencia a la tracción indirecta.

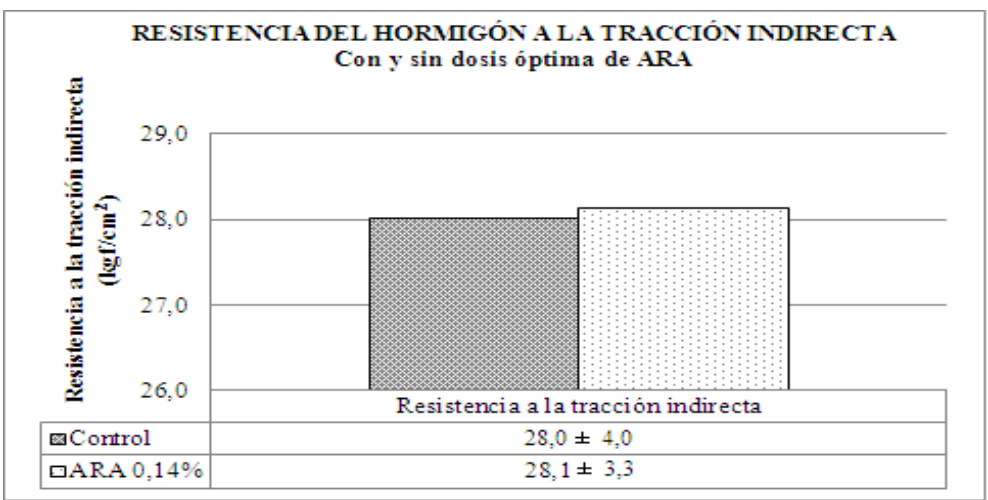

Figura 8: Resistencia del hormigón a la tracción indirecta con y sin dosis óptima de ARA.

La Figura 8 presenta la similitud en los valores de resistencia a la tracción indirecta que se obtuvo, las cuales, son de $28 \mathrm{kgf} / \mathrm{cm}^{2}$, cuya variabilidad es similar. Esto indica que la dosis óptima del ARA no afecta el comportamiento mecánico del hormigón la tracción indirecta.

\subsubsection{Resistencia a la tracción por flexión}

Siguiendo el procedimiento indicado en la ASTM C 78, se obtiene valores promedios del módulo de rotura de la muestra del hormigón de referencia y del hormigón con la dosis óptima de ARA. En la Figura 9 se presenta los valores donde se presenta que el módulo de rotura del hormigón de control es de 3,6\%. Los resultados indican que el comportamiento mecánico a la tracción por flexión no se altera significativamente al utilizar la dosis óptima de ARA.

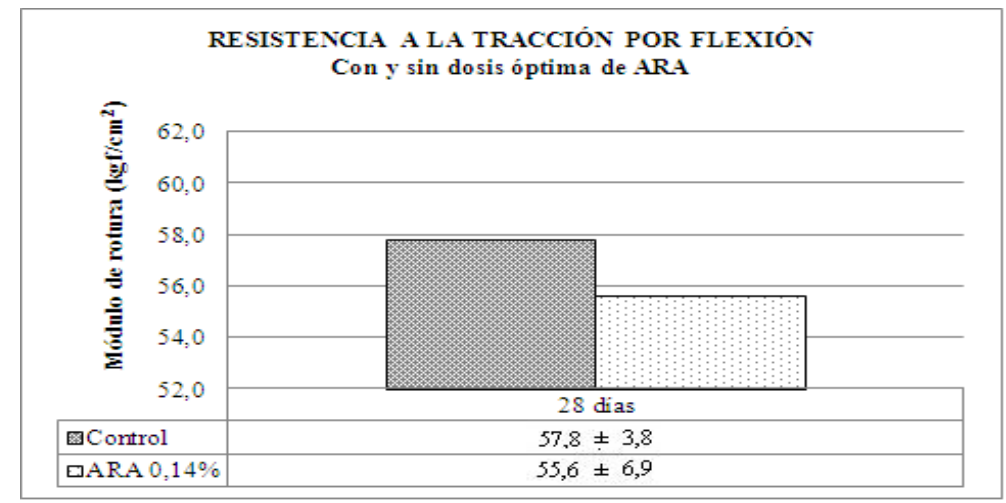

Figura 9: Módulo de rotura del hormigón de control y del hormigón con la dosis óptima de ARA. 


\subsubsection{Velocidad de pulso ultrasónico}

Al aplicar el procedimiento de la ASTM C 597, se empleó un aparato marca James Instruments, que está constituido por un generador de pulsos eléctricos de alta frecuencia, un transductor, un circuito receptor, un dispositivo de calibración y una unidad de lectura que permite registrar tiempos comprendidos en un rango mínimo de 20 microsegundos a 1000 microsegundos con aproximación del 1\%. Los resultados obtenidos indican que las velocidades promedios para ambos hormigones en estudio son iguales, lo que indica que la dosis óptima del ARA no afecta la homogeneidad del hormigón. En la Figura 10, se presentan las velocidades de pulso ultrasónico que alcanzan los $4,12 \mathrm{~km} / \mathrm{s}$, siendo este valor excelente para cualquier hormigón.

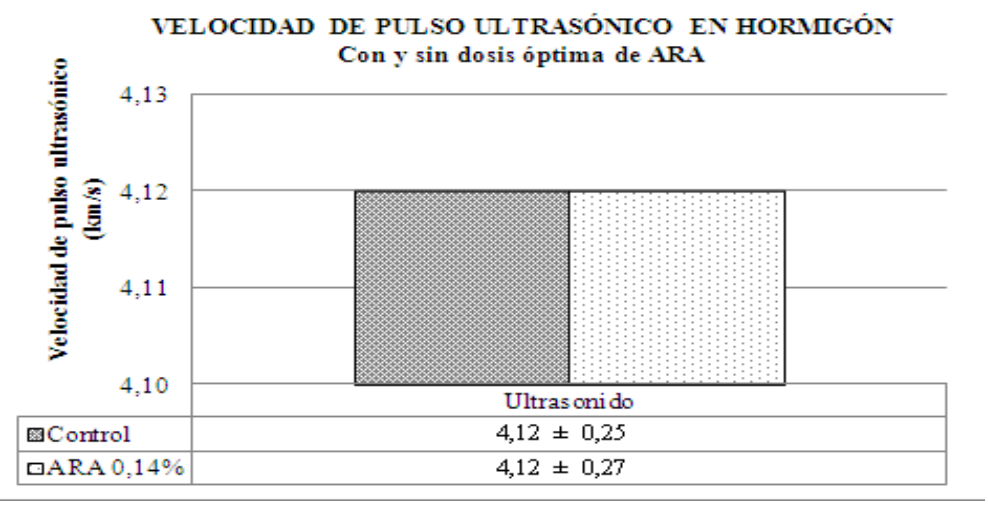

Figura 10: Velocidad promedio de pulso ultrasónico en probetas de hormigón con y sin ARA.

\subsubsection{Absorción del hormigón con y sin dosis óptima de ARA}

Los resultados obtenidos para la absorción del hormigón con y sin dosis óptima de ARA, una vez, aplicado el procedimiento de la ASTM C 642 se valida que no hay afectación desfavorable, ya que, los valores de absorción de ambos hormigones (control y dosis óptima de ARA) son similares 5,77\% y 5,74\%, respectivamente. Siendo menor la absorción para el hormigón con dosis óptima de ARA.

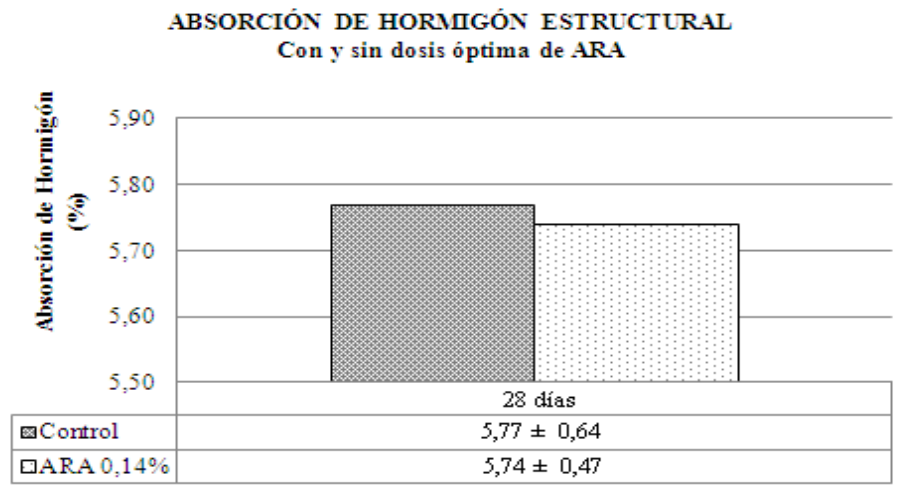

Figura 11: Absorción del hormigón estructural con y sin dosis óptima de ARA.

\subsubsection{Adherencia del acero de refuerzo y el hormigón.}

Los resultados obtenidos de la adherencia del acero de refuerzo con el hormigón, resultan interesantes, ya que, de 3 mezclas se obtuvieron 12 probetas por cada dosificación (control y dosis óptima de ARA), según procedimiento especificado en ASTM C 234. En la Figura 12 se presentan 2 fotografías, la primera donde se están preparando las probetas de hormigón con las barras de acero y, la segunda en el momento del ensayo de adherencia del acero de refuerzo con el hormigón, en la cual, se observa el aparato de medida del deslizamiento. 

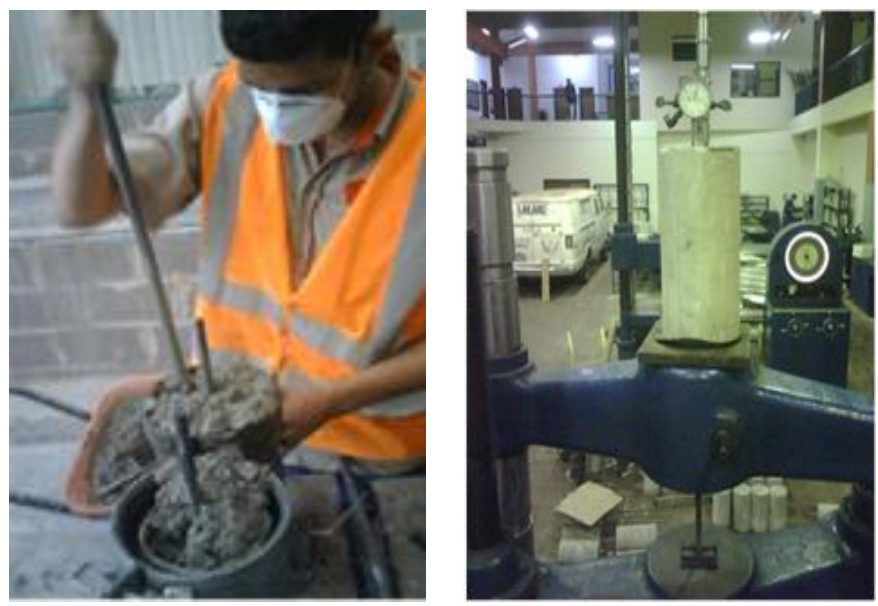

Figura 12: Fotografías a) Elaboración de probetas de adherencia; b) Ensayo de adherencia.

La Figura 13 presenta los resultados de resistencias promedio obtenidas para deslizar 0,10 mm, una barra de refuerzo adherida en el hormigón para ambas dosificaciones. Estos valores indican que la resistencia a la adherencia del hormigón con porcentaje óptimo de ARA $(0,14 \%$ por peso de cemento) es inferior en un $9 \%$ promedio respecto a la resistencia a la adherencia control. Es de interés resaltar que pasado el umbral del $5 \%$ de valores inferiores a la de control en esta prueba de mucha importancia para miembros estructurales, se afirma que el comportamiento mecánico de adherencia se ve afectado por la incorporación de la dosis óptima de ARA.

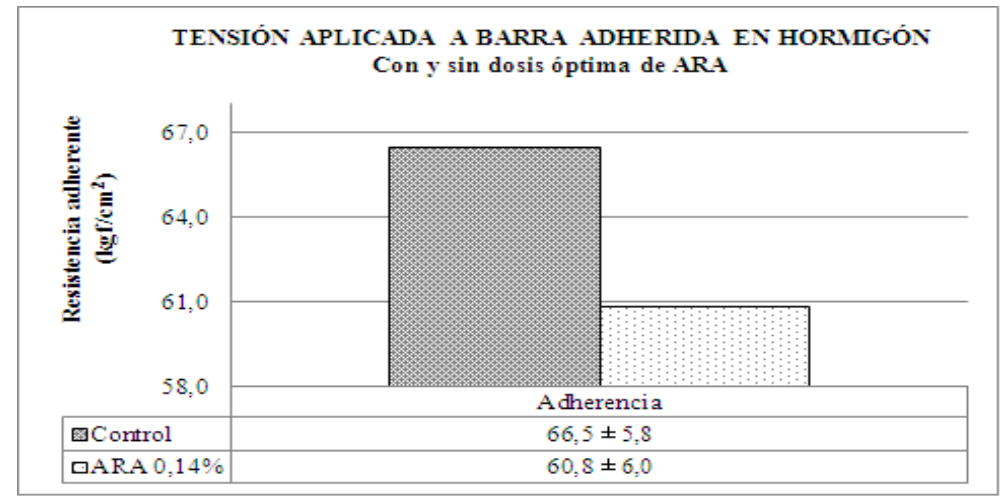

Figura 13: Tensión aplicada para deslizar $0,10 \mathrm{~mm}$ la barra adherida en el hormigón con y sin ARA.

\section{CONCLUSIONES}

El uso de ARA como aditivo es factible en las mezclas de hormigón no estructural, pudiendo ser utilizado en brocales, ornamentos, pavimentos rígidos, aceras, bloques, adoquines, morteros para hormigón ciclópeo y senderos peatonales. Esto se comprueba al incorporar la dosis óptima en el hormigón, el cual, posee comportamiento aceptable y similar al hormigón de control en los ensayos de asentamiento, tiempo de fraguado, absorción de agua, velocidad de pulso ultrasónico, resistencia a la compresión y a la tracción. El ensayo que no cumple es la adherencia desarrollada entre el hormigón y las barras de acero con resaltes, por ello, no es conveniente utilizar el ARA como aditivo en hormigón para miembros tipo vigas, columnas y losas.

\section{AGRADECIMIENTOS}

Los autores quieren agradecer a la planta mezcladora de hormigón perteneciente a la Fábrica Nacional de Cemento ubicada en San Antonio de El Valle, Caracas. Asimismo, al financiamiento realizado por el Consejo de Desarrollo Científico y Humanístico de la Universidad Central de Venezuela en el marco del proyecto de investigación $\mathrm{N}^{\circ}$ PSU-08-8843-2016/1. 


\section{BIBLIOGRAFÍA}

[1] EL-FADEL M., KHOURY R. "Strategies for vehicle waste-oil management: a case study". Resources, Conservation and Recycling, v.33, n. 2, pp- 75-91, Sep. 2001.

[2] TORRES J.,TROCHEZ J. Y MEJÍA R. "Reutilización de un residuo de la industria petroquímica como adición al cemento portland", Ingeniería y Ciencia, v 8, n. 15, pp.141-156, Jun. 2012.

[3] GARCÍA M., SÁNCHEZ M., FRÍAS M., MÚJIKA R. Comportamiento científico técnico de los cementos portland elaborados con catalizadores FCC, Monografía, Instituto Eduardo Torroja de las ciencias de la construcción, Madrid-España, 2006.

[4] PELLISER, F., ZAVARISE, N., LONGO, T., et al., "Concrete made with recycled tire rubber: Effect of alkaline activation and silica fume addition", Journal of cleaner production. Vol. 19, issues 6-7., pp. 757-763, April-may 2011

[5] TANGCHIRAPAT, W., SAETING, T., JATURAPITAKKUL, C., et al., "Use of waste ash from palm oil industry in concrete", Waste management, v. 27, n. 1, pp 81-88, 2007.

[6] PAPPU, A., SAXENA, M., ASOLEKAR, S. "Solid wastes generation in india and their recycling potencial in building materials", Building and environment, v. 42, n. 6, pp. 2311-2320, june 2007.

[7] FRIAS M., DE LA VILLA R., GARCÍA R., et al., "Mineralogical Evolution of Kaolin-Based Drinking Water Treatment Waste for Use as Pozzolanic Material:The Effect of Activation Temperature". Journal of the American Ceramic and Society, v.96, n.10, pp. 3188-3195, Oct. 2013.

[8] BILAL, S. H., AHMAD, A. R. "Effect of used engine oil on structural behavior of reinforced concrete elements”, Construction and Building Materials, v.17, n.17, pp. 203-211, May.2003.

[9] NASIR S., MUHD F., SALMIA B. (2011). "Properties of concrete containing used engine oil", International Journal of Sustainable Construction, Engineering \& Technology, v.2, n.1, pp. 72-82, Jun. 2011.

[10] PORRERO, J., RAMOS, C., GRÁSES, J., et al., Manual del concreto estructural. 4ta edición,. CaracasVenezuela, SIDETUR, 2012.

[11] GARCÍA, J. Método para la Dosificación de Hormigones. Publicación Técnica No 42, Instituto del Cemento Pórtland Argentino, Buenos Aires, 1964.

[12] RAMOS, G. Propuesta para determinar el comportamiento a la compresión en probetas cilindricas de $10 \mathrm{~cm} * 20 \mathrm{~cm}$ y $15 \mathrm{~cm} * 30 \mathrm{~cm}$ usadas para ensayos de resistencia a la compresión de concreto, Trabajo Especial de Grado, Instituto Universitario Politécnico Santiago Mariño, Caracas, Venezuela, 2007.

[13] BARRAGÁN, M., BOHÓRQUEZ, G. Estudio de factibilidad técnica y económica para la instalación de una planta de regeneración de lubricantes automotores en el Área Metropolitana. Trabajo Especial de Grado, Escuela de Procesos Químicos, Facultad de Ingeniería, Universidad Central de Venezuela, Caracas, Venezuela, 2000.

\section{ORCID}

Trino Baloa https://orcid.org/0000-0003-3376-9791

Juan De Abreu https://orcid.org/0000-0003-2568-4106

Carlos Arellano Sem ORCID

Idalberto Águila https://orcid.org/0000-0002-6621-9852 